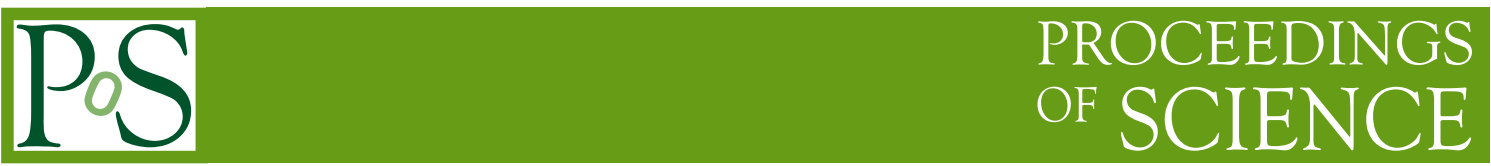

\title{
GravitinoPack and late decays involving gravitinos
}

\author{
Helmut Eberl \\ Institut für Hochenergiephysik der Österreichischen Akademie der Wissenschaften, \\ A-1050 Vienna, Austria \\ E-mail: helmut. eberleoeaw.ac.at \\ Vassilis C. Spanos* \\ University of Athens, Faculty of Physics, Department of Nuclear \& Particle Physics, \\ GR-15784 Athens, Greece \\ E-mail: vspanos@phys.uoa.gr
}

\begin{abstract}
In this talk, we present the package GravitinoPack that calculates decays of unstable supersymmetric particles, involving gravitinos in the final or initial state. If the gravitino is the dark matter particle and therefore stable, the package calculates the decays of the lightest neutralinos, and the lighter stau or stop NLSP into the gravitino LSP and one or two Standard Model particles. On the other hand, assuming that the gravitino is unstable, GravitinoPack calculates all its two-body and the three-body decay widths to the neutralino LSP and Standard Model particles. Since all these decays, involving the gravitino, are of gravitational nature, the lifetime of the decaying particle can be of the order of seconds are more, hence called "late decays". The precise knowledge of all the partial decay widths enables the user to apply accurately the relevant cosmological constraints.
\end{abstract}

18th International Conference From the Planck Scale to the Electroweak Scale 25-29 May 2015

Ioannina, Greece

${ }^{*}$ Speaker. 


\section{Introduction}

The supersymmetric partner of the graviton, the gravitino, belongs to the spectrum of models of particle physics, that incorporate the local version of supersymmetry, the so-called supergravity. Depending on the mass hierarchy of these models, in the case of $R$-parity conservation, the gravitino can be either the stable lightest supersymmetric particle (LSP) that plays the role of the dark matter (DM) particle, or it is heavier than the LSP and thus unstable.

If the gravitino $\widetilde{G}$ is the LSP, other supersymmetric particles as neutralinos and sfermions (e.g. stops or staus) are unstable and can decay into a gravitino and other Standard Model (SM) particles. These decays produce electromagnetic energy and hadrons which affect the primordial Big-Bang Nucleosynthesis (BBN) prediction [1] for the abundances of the light nuclei, like D, ${ }^{4} \mathrm{He},{ }^{3} \mathrm{He}$ and ${ }^{7} \mathrm{Li}[2,3,4]$. On the other hand if the gravitino is not stable it can decay to the lightest neutralino $\left(\tilde{\chi}_{1}^{0}\right)$, that is the LSP in this case, and other SM particles.

For both cases we have presented results, for the unstable [5] and the stable [6] gravitino, using the software GravitinoPack [6]. This package is a numerical tool which we developed with the help of the packages FeynArts (FA), that was extended in order to deal with interactions with spin-3/2 particles with the particles of the Minimal Supersymmetric Standard Model (MSSM), and FormCalC (FC) [7, 8, 9], by using the Weyl-van-der-Waerden formalism [10] as implemented into FC from [11]. GravitinoPack contains ForTRAN77 and MATHEMATICA routines that calculate the decay widths for the relevant decay channels.

In [5] we studied in detail all dominant two-body channels $\widetilde{G} \rightarrow \widetilde{X} Y$, as well as the threebody channels $\widetilde{G} \rightarrow \tilde{\chi}_{1}^{0} X Y$, where $\widetilde{X}$ is a sparticle, $\tilde{\chi}_{1}^{0}$ the lightest neutralino and $X, Y$ are SM particles. The two-body decays dominate the total gravitino width, and in particular the channel $\widetilde{G} \rightarrow \tilde{\chi}_{1}^{0} \gamma$, which is kinematically open in the whole region $m_{\widetilde{G}}>m_{\tilde{\chi}_{1}^{0}}$. On the other hand, also many three-body decay channels can be open, $\widetilde{G} \rightarrow \tilde{\chi}_{1}^{0} X Y$, even below thresholds of involved two-body decays, $m_{\widetilde{G}}<m_{\widetilde{X}}+m_{Y}$. For the gravitino DM models (stable gravitino) the Next to the Lightest Supersymmetric Particle (NLSP) can be the lightest neutralino $\left(\tilde{\chi}_{1}^{0}\right)$, or the lighter stau $\left(\tilde{\tau}_{1}\right)$, or the lighter stop $\left(\tilde{t}_{1}\right)$. In this cases GravitinoPack calculates all two-body decay channels e. g. the dominant channel $\tilde{\chi}_{1}^{0} \rightarrow \widetilde{G} \gamma$, or $\tilde{\tau}_{1} \rightarrow \widetilde{G} \tau$ or $\tilde{t}_{1} \rightarrow \widetilde{G} t$, as well as all possible three-body decays.

As numerical applications of the package we study a few representative benchmark points from supersymmetric models with different supersymmetry breaking patterns, like the phenomenological MSSM (pMSSM) [12] and the Constrained MSSM (CMSSM) [13]. For the pMSSM especially, we have selected points where the neutralino carries significant Higgsino components as in the Non-Universal Higgs Model (NUHM) [14].

\section{The Decays in the GravitinoPack}

The main aspects were already presented in [5] and in the Appendix of [6]. There, one can find a detailed derivation of the 78 gravitino couplings with the MSSM particles. Furthermore, in [6] also the manual of GravitinoPack is included. The partial widths of all decay channels presented in this section can be calculated with GravitinoPack. 


\subsection{Two-body decays of $\widetilde{G}$}

The gravitino decays into a fermion $F$, and a scalar $S$ or a vector particle $V$ are

$$
\begin{aligned}
\widetilde{G} & \rightarrow F S \\
& \rightarrow f \tilde{f}_{i}^{*}, \bar{f} \tilde{f}_{i}, \tilde{\chi}_{j}^{0}\left(h^{0}, H^{0}, A^{0}\right), \tilde{\chi}_{k}^{+} H^{-}, \tilde{\chi}_{k}^{-} H^{+}, \\
\widetilde{G} & \rightarrow F V \\
& \rightarrow \tilde{g} g, \tilde{\chi}_{j}^{0}\left(Z^{0}, \gamma\right), \tilde{\chi}_{k}^{+} W^{-}, \tilde{\chi}_{k}^{-} W^{+},
\end{aligned}
$$

where $f$ denotes a SM fermion, $f=v_{e}, v_{\mu}, v_{\tau}, e^{-}, \mu^{-}, \tau^{-}, u, c, t, d, s, b$. Its corresponding superpartners are the sfermions $\tilde{f}_{i}, i=1,2$. The four neutralino states are $\tilde{\chi}_{j}^{0}, j=1, \ldots, 4$ and the two charginos $\tilde{\chi}_{k}^{ \pm}, k=1,2$. With $g$ we denote the gluon and with $\tilde{g}$ its superpartner, the gluino. Furthermore, in the MSSM we have three neutral Higgs bosons (two $C P$-even: $h^{0}$ and $H^{0}$, and one $C P$-odd: $A^{0}$ ) and two charged Higgs bosons: $H^{ \pm}$. The vector bosons are the photon $\gamma$, the $Z$-boson $Z^{0}$ and the $W$-bosons $W^{ \pm}$.

\subsection{Three-body decays of $\widetilde{G}$}

The gravitino decays into a neutralino and a pair of SM particles are

$$
\begin{aligned}
& \widetilde{G} \rightarrow \tilde{\chi}_{i}^{0} \bar{f} f, \\
& \widetilde{G} \rightarrow \tilde{\chi}_{i}^{0} V V, \quad V V=Z^{0} Z^{0}, Z^{0} \gamma, W^{+} W^{-}, \\
& \widetilde{G} \rightarrow \tilde{\chi}_{i}^{0} V S, \quad V S=\left(Z^{0}, \gamma\right)\left(h^{0}, H^{0}, A^{0}\right), W^{+} H^{-}, W^{-} H^{+}, \\
& \widetilde{G} \rightarrow \tilde{\chi}_{i}^{0} S S, \quad S S=\left(h^{0}, H^{0}, A^{0}\right)\left(h^{0}, H^{0}, A^{0}\right), H^{+} H^{-} .
\end{aligned}
$$

These are 19 three-body decay channels. Note, that $\widetilde{G} \rightarrow \tilde{\chi}_{i}^{0} W^{-} H^{+}$and its charge conjugated process $\widetilde{G} \rightarrow \tilde{\chi}_{i}^{0} W^{+} H^{-}$count as individual contributions, but $\Gamma\left(\widetilde{G} \rightarrow \tilde{\chi}_{i}^{0} W^{-} H^{+}\right)=\Gamma\left(\widetilde{G} \rightarrow \tilde{\chi}_{i}^{0} W^{+} H^{-}\right)$.

We use as an example the process $\widetilde{G} \rightarrow \tilde{\chi}_{i}^{0} W^{-} W^{+}$to illustrate the nine individual amplitudes contributing to this channel, as plotted in Figure 1.

Now we turn to the stable gravitino scenario, discussing the decays of the lightest neutralino $\widetilde{\chi}_{1}^{0}$ to gravitino and SM particles, followed by the discussion on the stau and stop NLSP decays.

\section{$2.3 \widetilde{\chi}_{1}^{0}$ decays into $\widetilde{G}$}

Five two-body decays are possible:

$$
\begin{aligned}
& \widetilde{\chi}_{1}^{0} \rightarrow \widetilde{G}\left(Z^{0}, \gamma\right), \\
& \widetilde{\chi}_{1}^{0} \rightarrow \widetilde{G}\left(h^{0}, H^{0}, A^{0}\right) .
\end{aligned}
$$

The lightest neutralino can decay into the gravitino and a pair of SM particles as

$$
\begin{aligned}
& \widetilde{\chi}_{1}^{0} \rightarrow \widetilde{G} \bar{f} f, \\
& \widetilde{\chi}_{1}^{0} \rightarrow \widetilde{G} V V, \quad V V=Z^{0} Z^{0}, Z^{0} \gamma, W^{+} W^{-}, \\
& \widetilde{\chi}_{1}^{0} \rightarrow \widetilde{G} V S, \quad V S=\left(Z^{0}, \gamma\right)\left(h^{0}, H^{0}, A^{0}\right), W^{+} H^{-}, W^{-} H^{+}, \\
& \widetilde{\chi}_{1}^{0} \rightarrow \widetilde{G} S S, \quad S S=\left(h^{0}, H^{0}, A^{0}\right)\left(h^{0}, H^{0}, A^{0}\right), H^{+} H^{-} .
\end{aligned}
$$

They are the same as given in eq. (2.2) with neutralino and gravitino interchanged. 


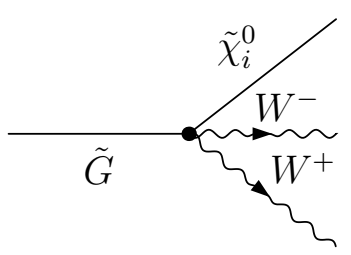

a)

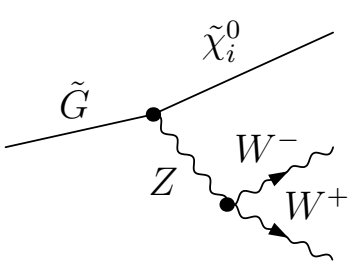

e)

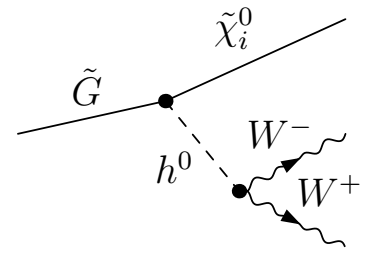

b)

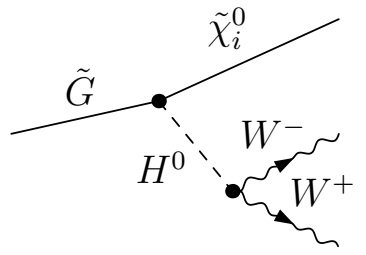

c)

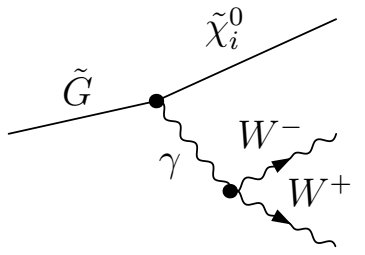

d)

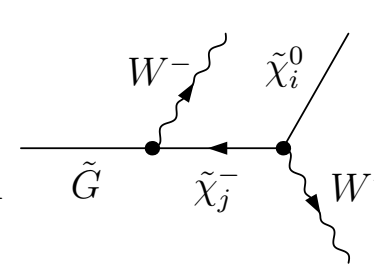

f)

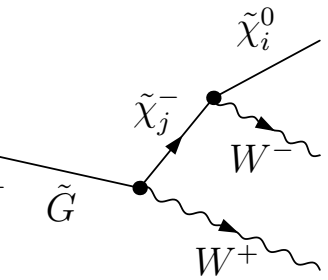

g)

Figure 1: Feynman graphs for the decay $\widetilde{G} \rightarrow \tilde{\chi}_{i}^{0} W^{-} W^{+}$.

\section{$2.4 \tilde{\tau}_{1}^{-}$decays into $\widetilde{G}$}

The only possible stau NLSP two-body decay is

$$
\tilde{\tau}_{1}^{-} \rightarrow \widetilde{G} \tau^{-} .
$$

The corresponding three-body decays are

$$
\begin{aligned}
& \tilde{\tau}_{1}^{-} \rightarrow \widetilde{G} Z^{0} \tau^{-}, \\
& \tilde{\tau}_{1}^{-} \rightarrow \widetilde{G} W^{-} v_{\tau}, \\
& \tilde{\tau}_{1}^{-} \rightarrow \widetilde{G}\left(h^{0}, H^{0}, A^{0}\right) \tau^{-}, \\
& \tilde{\tau}_{1}^{-} \rightarrow \widetilde{G} H^{-} v_{\tau} .
\end{aligned}
$$

\section{$2.5 \tilde{t}_{1}$ decays into $\widetilde{G}$}

There is only one stop NLSP two-body decay possible,

$$
\tilde{t}_{1} \rightarrow \widetilde{G} t .
$$

The possible three-body decays of $\tilde{t}_{1}$ are

$$
\begin{aligned}
\tilde{t}_{1} & \rightarrow \widetilde{G} Z^{0} t, \\
\tilde{t}_{1} & \rightarrow \widetilde{G} W^{+} b, \\
\tilde{t}_{1} & \rightarrow \widetilde{G}\left(h^{0}, H^{0}, A^{0}\right) t, \\
\tilde{t}_{1} & \rightarrow \widetilde{G} H^{+} b .
\end{aligned}
$$

\section{Numerical results}

In our numerical analysis we choose representative points from various supersymmetric models, assuming different mechanism for the supersymmetry breaking. In particular, we study points 

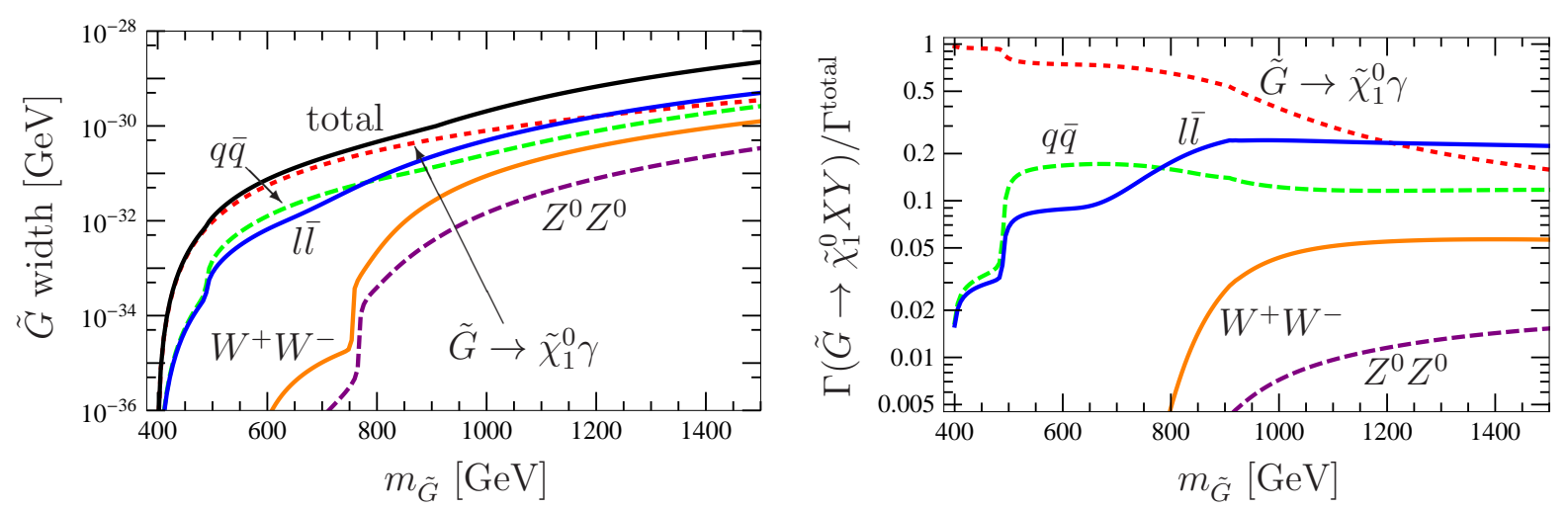

Figure 2: The three-body decay widths of the gravitino decaying into $\tilde{\chi}_{1}^{0}$ and $q \bar{q}, l \bar{l}, W$-pairs, and $Z$-pairs at the $A^{0}$-funnel point; "total" denotes $\Gamma^{\text {total }}$ which is the full two-body width plus the sum of the non-resonant part of three-body decay widths with $\tilde{\chi}_{1}^{0} ; q \bar{q}$ stands for the sum over all six quark flavours and $l \bar{l}$ for the sum over the three charged lepton and three neutrino flavours. The red dotted lines denote the two-body decay $\widetilde{G} \rightarrow \tilde{\chi}_{1}^{0} \gamma$. In the right figure we display the corresponding branching ratios for the decay channels plotted in the left figure.

based on the pMSSM [12] and in the CMSSM [13]. The benchmark points we study in this section are compatible with the cosmological $[15,16]$ and LHC constraints (Higgs mass $\simeq 125 \mathrm{GeV}$, LHCb bounds for rare decays etc.) $[17,18]$.

Our first numerical example is in the context of neutralino DM models with unstable gravitino, using a pMSSM point with the SUSY parameters chosen as $\tan \beta=20, \mu=700 \mathrm{GeV}, M_{A}=$ $770 \mathrm{GeV},\left(M_{1}, M_{2}, M_{3}\right)=(400,800,2400) \mathrm{GeV}, A_{t}=A_{b}=-2050 \mathrm{GeV}, A_{\tau}=-1000 \mathrm{GeV}, m_{\widetilde{q}_{L}}=$ $m_{\widetilde{u}_{R}}=m_{\widetilde{d}_{R}}=m_{\widetilde{b}_{R}}=1500 \mathrm{GeV}, m_{\widetilde{\ell}_{L}}=600 \mathrm{GeV}, m_{\widetilde{e}_{R}}=2500 \mathrm{GeV}, m_{\widetilde{Q}_{3 L}}=m_{\widetilde{L}_{3 L}}=800 \mathrm{GeV}$ and $m_{\widetilde{\tau}_{R}}=2000 \mathrm{GeV}$. In this point the neutralino relic density lies cosmologically along the so-called $A^{0}$-funnel region. Thus, we show in Figure 2 the three-body decay widths of the gravitino decaying into $\tilde{\chi}_{1}^{0}$ together with $q \bar{q}, l \bar{l}$, and $W, Z$-boson pairs there. We also show the two-body decay channel $\widetilde{G} \rightarrow \tilde{\chi}_{1}^{0} \gamma$ as a reference, because it is the dominant one for small $m_{\widetilde{G}}$. In the left figure we also show $\Gamma^{\text {total }}$ which is the full two-body width plus the sum of the non-resonant part of three-body decay widths with $\tilde{\chi}_{1}^{0}$, denoted by "total". In the right figure we show the relative quantities in terms of $\Gamma^{\text {total }} ; q \bar{q}$ stands for the sum over all six quark flavours, $\sum_{i=1,6} \Gamma^{\text {reso }+ \text { non-reso }}\left(\widetilde{G} \rightarrow \tilde{\chi}_{1}^{0} q_{i} \bar{q}_{i}\right)$ and $l \bar{l}, q_{i}=u, d, c, s, t, b$, and $l \bar{l}$ the sum of the three charged lepton and three neutrino flavours, $\sum_{j=1,3}\left(\Gamma^{\text {non }- \text { reso }+\operatorname{reso}}\left(\widetilde{G} \rightarrow \tilde{\chi}_{1}^{0} l_{j}^{+} l_{j}^{-}\right)+\Gamma^{\text {non-reso }+\operatorname{reso}}\left(\widetilde{G} \rightarrow \tilde{\chi}_{1}^{0} v_{l_{j}} \bar{v}_{l_{j}}\right)\right), l_{j}=e, v, \tau$. The decay width summing up the decays into all fermion pair can reach $38 \%$, into the $W$-boson pair $5.6 \%$ and into the $Z$-boson pair $1.5 \%$ in the shown range. The analogous plots for the coannhilation point look similar but the decays into $W$ - and $Z$-boson pairs are suppressed by about two orders of magnitude due to the pure bino state of the LSP.

The second numerical example we discuss is based on a model with stable gravitino (GDM) in the CMSSM We use $m_{0}=1600, M_{1 / 2}=5000, A_{0}=-4000 \mathrm{GeV}$, and $\tan \beta=10$. The mass of $\tilde{\chi}_{1}^{0}$ is $2282 \mathrm{GeV}$. It is worth mentioning that the gravitino DM relic density and the NLSP relic density are related by

$$
\frac{\Omega_{\mathrm{NLSP}}}{\Omega_{\widetilde{G}}}=\frac{m_{\mathrm{NLSP}}}{m_{\widetilde{G}}}>1
$$



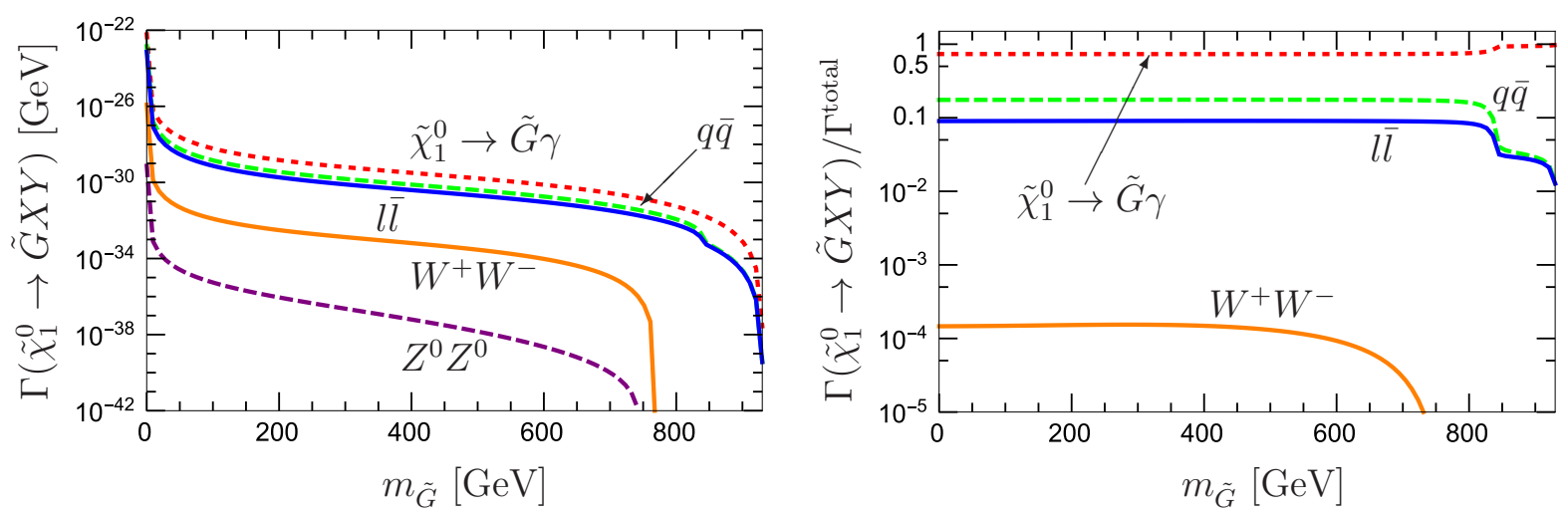

Figure 3: The three-body decay widths of the neutralino NLSP decaying into the gravitino $\widetilde{G}$ and other particles, in the GDM/CMSSM scenario. The dominant channels $q \bar{q}, l \bar{l}, W^{+} W^{-}$, and $Z Z$ are marked in the figure; $q \bar{q}$ stands for the sum over all six quark flavours and $l \bar{l}$ for the sum over the three charged lepton and three neutrino flavours. The red dotted lines denote the two-body decay $\tilde{\chi}_{1}^{0} \rightarrow \widetilde{G} \gamma$. In the right figure we display the corresponding branching ratios for the decay channels plotted in the left figure.

The cosmological bound for the gravitino relic density can be understood as upper bound $\Omega_{\widetilde{G}} h^{2} \lesssim$ 0.119. Therefore, one can have in addition gravitino production during reheating after inflation, if the reheating temperature is relatively large, of the order of $\sim 10^{10} \mathrm{GeV}$.
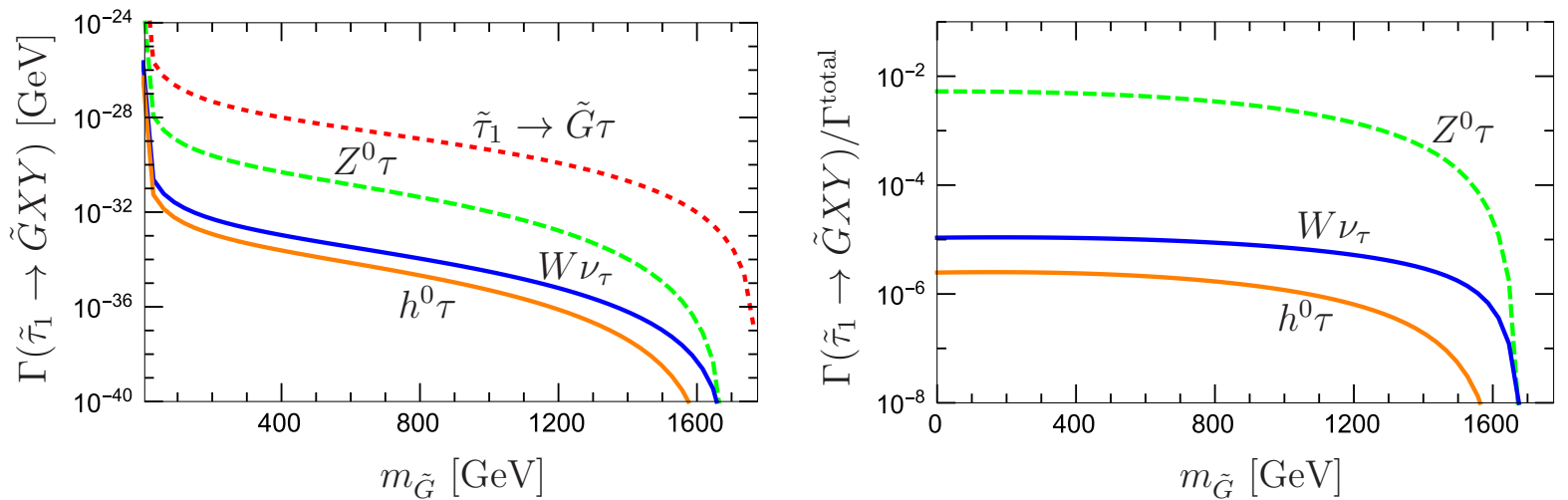

Figure 4: The three-body decay widths of the stau NLSP decaying into the gravitino $\widetilde{G}$ and other SM particles, in the GDM/CMSSM scenario. We present the dominant two-body channel $\widetilde{G} \tau$ and the three-body channels $\widetilde{G} Z \tau, \widetilde{G} W^{-} v_{\tau}$ and $\widetilde{G} h \tau$. In the right figure we display the corresponding branching ratios for the decay channels plotted in the left figure except $\tilde{\tau}_{1} \rightarrow \widetilde{G} \tau$.

In Figure 3 we present the corresponding decay widths (left figure) and the branching ratios (right figure) for the neutralino decays into $\widetilde{G}$ and other particles. The dominant channels $q \bar{q}, l \bar{l}$, $W^{+} W^{-}$, and $Z Z$ are marked in the figure; $q \bar{q}$ stands for the sum over all six quark flavours and $l \bar{l}$ for the sum over the three charged lepton and three neutrino flavours. The red dotted lines denote the two-body decay $\tilde{\chi}_{1}^{0} \rightarrow \widetilde{G} \gamma$, that dominates the neutralino decay amplitude, as can be seen in the left panel that illustrates the branching ratios. On the other hand, the three-body decay channels $q \bar{q}$ and $l \bar{l}$ are of the order of $10 \%$, while the $W^{+} W^{-}$, and $Z Z$ channels are much smaller. For this particular CMSSM point the other decay channels are even smaller. This happens because the neutralino is 

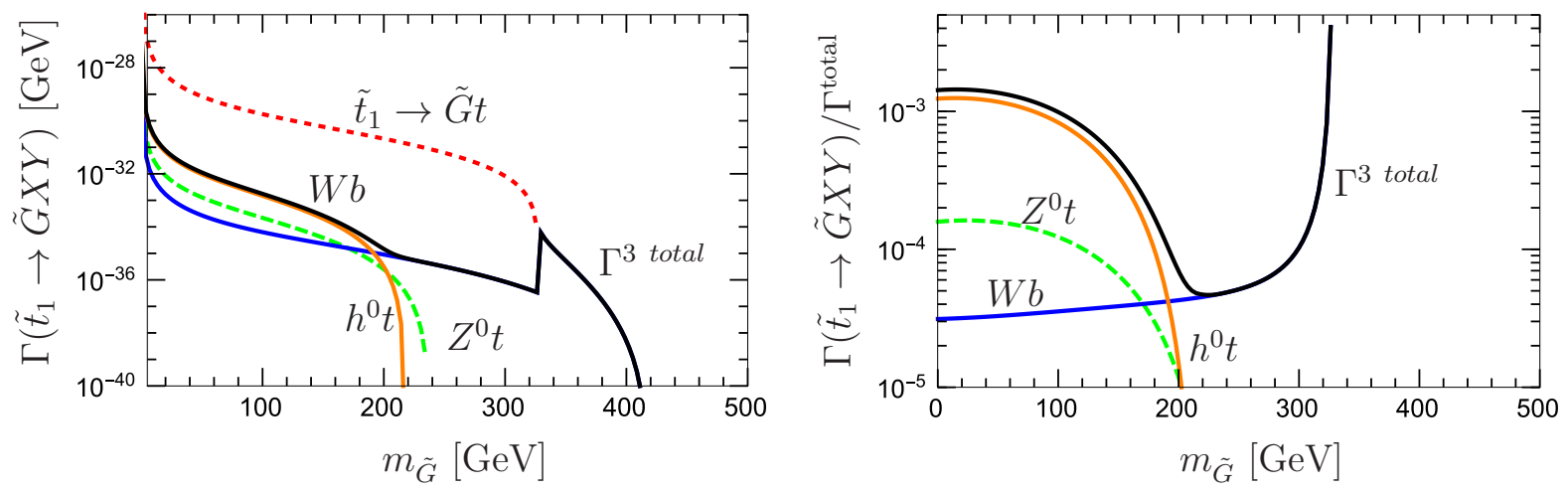

Figure 5: The three-body decay widths of the stop NLSP decaying into the gravitino $\widetilde{G}$ and other SM particles, in the GDM/CMSSM scenario. We present the dominant two-body channel $\widetilde{G} \tau$ and the three-body channels $\widetilde{G} Z t, \widetilde{G} W^{-} b$ and $\widetilde{G} h^{0} b$. In the right figure we display the corresponding branching ratios for the decay channels plotted in the left figure except $\tilde{t}_{1} \rightarrow \widetilde{G} t$.

predominantly a bino at this particular point of the parameter space.

In Figure 4 we present the decays widths (left figure) and the branching ratios (right figure) for the stau NLSP decays into the gravitino and other particles, in the GDM scenario. The CMSSM parameters are $m_{0}=1000, M_{1 / 2}=4200, A_{0}=-2500 \mathrm{GeV}$, and $\tan \beta=10$. The mass of $\tilde{\tau}_{1}$ is 1795 $\mathrm{GeV}$. The dominant decay channel is the two-body decay $\tilde{\tau}_{1} \rightarrow \widetilde{G} \tau$. In addition we plot the threebody channels $\tilde{\tau}_{1} \rightarrow \widetilde{G} Z^{0} \tau, \tilde{\tau}_{1} \rightarrow \widetilde{G} W^{-} v_{\tau}$, and $\tilde{\tau}_{1} \rightarrow \widetilde{G} h^{0} \tau$. The widths of the channels involving heavier Higgs bosons in the final state, are much smaller or even zero.

Similarly, in Figure 5 we present the decays widths (left figure) and the branching ratios (right figure) for the stop NLSP decays into the gravitino $\widetilde{G}$ and other particles. The CMSSM parameters are $m_{0}=3000, M_{1 / 2}=1090, A_{0}=-7500 \mathrm{GeV}$ and $\tan \beta=30$. The mass of the NLSP $\tilde{t}_{1}$ is $501 \mathrm{GeV}$. In addition to $\tilde{t}_{1} \rightarrow \widetilde{G} t$ we present also the three-body decay channels into $\widetilde{G} Z^{0} t, \widetilde{G} W^{-} b$ and $\widetilde{G} h^{0} t$. Again, the channels involving the heavier Higgs bosons are negligible. The dominant decay channel is $\tilde{t}_{1} \rightarrow \widetilde{G} t$, up to the kinematical threshold $m_{\widetilde{G}}=m_{\tilde{t}_{1}}-m_{t} \sim 328 \mathrm{GeV}$. As can been seen in both plots in Figure 5, beyond this point the two-body channel is closed and it dominates the three-body channel $\tilde{t}_{1} \rightarrow \widetilde{G} W^{-} b$. This is clearer visible in the right plot, where for $m_{\widetilde{G}}>m_{\tilde{t}_{1}}-m_{t}$ the $\widetilde{G} W^{-} b$ decay channel grows after this point and eventually reach the maximum value one outside of the displayed region. Recently, a paper studying in particular, this three-body channel appeared [19].

In summary, we have discussed representative cases both in the pMSSM and CMSSM. Based on these examples one can see that the full knowledge of the two- and three-body decay channels of the NLSP is essential for the precise calculation of the decay width and the various branching ratios. This is actually the big advantage of using GravitinoPack, since it gives all computed results both in FORTRAN77 and within the MATHEMATICA environment. It also supports SLHA input format. 


\section{Summary}

We have studied supersymmetric models, where the gravitino is either unstable or stable. If it is unstable it can decay to the neutralino LSP and SM particles. If it is the stable LSP it can play the role of the DM particle and the NLSP can be the lightest neutralino $\tilde{\chi}_{1}^{0}$ or a sfermion, as stau $\tilde{\tau}_{1}$ or stop $\tilde{t}_{1}$.

In this talk we have discussed all these cases using the recently presented public available computer tool GravitinoPack. This numerical package based on an auto-generated FORTRAN77 code, calculates the branching ratios and decay widths for the NSLP decays, if the gravitino is stable and the DM particle. In this case we have calculated decays of the NLSP neutralino, stau, and stop to the gravitino LSP and one or two SM particles. We have also discussed the complementary case, where the gravitino is unstable and can decay into the neutralino LSP and SM particles. The products of these decays carry electromagnetic energy and can build hadrons that influence the predictions of BBN, since the gravitational nature of these decays place them in this time scale. Therefore, the detailed knowledge of the relevant branching ratios and decay widths is important to study and constrain various supergravity models.

\section{Acknowledgements}

This work is supported by the Austrian Science Fund (FWF) P 26338-N27 and by the European Commission through the "HiggsTool" Initial Training Network PITN-GA-2012-316704.

\section{References}

[1] D. Lindley, Astrophys. J. 294 (1985) 1; J. R. Ellis, D. V. Nanopoulos and S. Sarkar, Nucl. Phys. B 259 (1985) 175; D. Lindley, Phys. Lett. B 171 (1986) 235; M. H. Reno and D. Seckel, Phys. Rev. D 37 (1988) 3441; S. Dimopoulos, R. Esmailzadeh, L. J. Hall and G. D. Starkman, Astrophys. J. 330, 545 (1988); J. R. Ellis, G. B. Gelmini, J. L. Lopez, D. V. Nanopoulos and S. Sarkar, Nucl. Phys. B 373 (1992) 399.

[2] M. Kawasaki and T. Moroi, Prog. Theor. Phys. 93 (1995) 879 [arXiv:hep-ph/9403364]; M. Kawasaki, K. Kohri and T. Moroi, Phys. Rev. D 63 (2001) 103502 [arXiv:hep-ph/0012279].

[3] R. H. Cyburt, J. R. Ellis, B. D. Fields and K. A. Olive, Phys. Rev. D 67 (2003) 103521 [arXiv:astro-ph/0211258]; K. Jedamzik, Phys. Rev. D 70 (2004) 063524 [arXiv:astro-ph/0402344].

[4] R. H. Cyburt, J. Ellis, B. D. Fields, F. Luo, K. A. Olive and V. C. Spanos, JCAP 0910 (2009) 021 [arXiv:0907.5003 [astro-ph.CO]]; R. H. Cyburt, J. Ellis, B. D. Fields, F. Luo, K. A. Olive and V. C. Spanos, arXiv:1303.0574 [astro-ph.CO]. N. E. Mavromatos and V. C. Spanos, Phys. Rev. D 87 (2013) 035025 [arXiv:1212.6386 [hep-ph]].

[5] H. Eberl and V. C. Spanos, JHEP 1308 (2013) 055 [arXiv:1305.6934 [hep-ph]].

[6] H. Eberl and V. C. Spanos, arXiv:1509.09159 [hep-ph].

[7] T. Hahn, Comput. Phys. Commun. 140 (2001) 418 [arXiv:hep-ph/0012260].

[8] T. Hahn and C. Schappacher, Comput. Phys. Commun. 143 (2002) 54 [arXiv:hep-ph/0105349].

[9] T. Hahn and M. Perez-Victoria, Comput. Phys. Commun. 118 (1999) 153 [hep-ph/9807565]. 
[10] H. Weyl, The Theory of Groups and Quantum Mechanics (Dover, New York, 1931); B.L. van der Waerden, Group Theory and Quantum Mechanics (Springer-Verlag, Berlin, 1974).

[11] S. Dittmaier, Phys. Rev. D 59 (1998) 016007 [hep-ph/9805445].

[12] A. Djouadi et al. [MSSM Working Group Collaboration], hep-ph/9901246.

[13] M. Drees and M. M. Nojiri, Phys. Rev. D 47 (1993) 376 [hep-ph/9207234]; G. L. Kane, C. F. Kolda, L. Roszkowski and J. D. Wells, Phys. Rev. D 49 (1994) 6173 [hep-ph/9312272]; H. Baer and M. Brhlik, Phys. Rev. D 53 (1996) 597 [hep-ph/9508321]; J. R. Ellis, T. Falk, K. A. Olive and M. Schmitt, Phys. Lett. B 388 (1996) 97 [hep-ph/9607292]; A. B. Lahanas, D. V. Nanopoulos and V. C. Spanos, Phys. Rev. D 62 (2000) 023515 [hep-ph/9909497]. L. Roszkowski, R. Ruiz de Austri and T. Nihei, JHEP 0108 (2001) 024 [hep-ph/0106334]; U. Chattopadhyay, A. Corsetti and P. Nath, Phys. Rev. D 66 (2002) 035003 [hep-ph/0201001]; J. R. Ellis, K. A. Olive, Y. Santoso and V. C. Spanos, Phys. Lett. B 565 (2003) 176 [hep-ph/0303043]; M. Argyrou, A. B. Lahanas and V. C. Spanos, JHEP 0805 (2008) 026 [arXiv:0804.2613 [hep-ph]].

[14] P. Nath and R. L. Arnowitt, Phys. Rev. D 56 (1997) 2820 [hep-ph/9701301]; H. Baer, A. Mustafayev, S. Profumo, A. Belyaev and X. Tata, Phys. Rev. D 71 (2005) 095008 [hep-ph/0412059]; J. R. Ellis, K. A. Olive and P. Sandick, Phys. Rev. D 78 (2008) 075012 [arXiv:0805.2343 [hep-ph]].

[15] P. A. R. Ade et al. [Planck Collaboration], arXiv:1502.01589 [astro-ph.CO].

[16] E. Aprile et al. [XENON100 Collaboration], Phys. Rev. Lett. 109 (2012) 181301 [arXiv:1207.5988 [astro-ph.CO]].

[17] W. Porod, Comput. Phys. Commun. 153 (2003) 275 [hep-ph/0301101]; W. Porod and F. Staub, Comput. Phys. Commun. 183 (2012) 2458 [arXiv:1104.1573 [hep-ph]].

[18] M. Frank, T. Hahn, S. Heinemeyer, W. Hollik, H. Rzehak and G. Weiglein, JHEP 0702 (2007) 047 [hep-ph/0611326]; G. Degrassi, S. Heinemeyer, W. Hollik, P. Slavich and G. Weiglein, Eur. Phys. J. C 28 (2003) 133 [hep-ph/0212020].

[19] J. Lorenzo Díaz Cruz and Bryan Larios López, arXiv:1510.01447 [hep-ph]. 